glücklichem. letaleın Ausgang mit den dazu gehörigen Obduktionsbefunden beobachtet worden.

Ein anderer geg enüber den unlöslichen Quecksilberpräparaten erhobener Vorwurf basirt auf der klinischen Wahrnehmung, dass, falls die injizirte Flüssigkeit in eine Vene gelangt, eine Lungenembolie mit eventuell nachfolgendem Lungeninfarkt zu Stande kommen kann, eine Thatsache, die unter allen Umständen eine gewisse Gefahr in sich birgt, auch wenn es in der Mehrzahl der Fälle ohne ernstere Folgen abgeht.

Eine weitere Unannehmlichkeit besteht darin, dass eine grosse Anzahl von Patienten neben allgemeinen körperlichen Störungen auf die Injektionen mit mehr oder minder gros sen Schmerzen am Orte der Applikation reagirt, dass die Einspritzungen von theilweise recht $\mathrm{sch}$ merzhaften Infiltraten gefolgt sein können, die ihrerseits kürzere oder längere Zeit dauern, oft Jahre hindurch ganz symptomlos fortbestehen, unter Umständen aber auch, wie die Erfahrung gelehrt hat, schliesslich in Abszessen, d. h. zumeist aseptischen, chemisch bedingten Erweichungsheerden ihren Ausgang finden.

Es kann hier nicht unsere Aufgabe sein, darüber zu disku-

\section{Kasuistischer Beitrag zur Genese der Gummata syphilitica.')}

\author{
Von Dr. G. Kulisch in Halle a. S.
}

Wenn man einen Rückblick auf die Syphilislitteratur delletzten vier Jahrzehnte wirft, so nimmt in derselben das Studium der verschiedenen Methoden der Quecksilberdarreichung, resp. Einverleibung einen nicht unbedeutenden Raum ein.

Während, wie ja allgemein bekannt, von dem ersten epidemischen Auftreten der Syphilis zu Ende des 15. Jahrhunderts an, also etwa vier Jahrhunderte hindurch die Inunktionskur mittels Quecksilbersalbe trotz vielfacher Anfechtungen und therapeutischer Ersatzvorschläge immer wieder die Oberhand gewonnen und schliesslich das Feld behauptet hatte - weil eben mit ihrer Hilfe immer noch die besten und zuverlässigsten Resultate erzielt wurden -, erstand in den sechziger Jahren des vorigen Jahrhunderts der Einreibungskur in der Methode der subkutanen Einverleibung von Quecksilberpräparaten eine scharfe Conkurrentin.

Es war dies umsoweniger zu verwundern, als der Inunktionskur - einer von Hause aus roh-empirischen, unwissenschaftlichen Methode - nicht nur vom ästhetischen, sondern auch vom praktischen und wissenschaftlichen Standpunkte aus gewisse Mängel anhafteten, in letzterer Beziehung ihr gegenüber vor allem, und zwar mit Fug und Recht, immer wieder der Vorwurf erhoben wurde, dass man absolut kein sicheres Urtheil iiber die Form und Menge, in welcher das Quecksilber dem Organismus einverleibt werde, abzugeben im Stande sei.

Im Laufe der Jahre kamen dann sowohl experimentell als auch praktisch-klinisch eine Reihe von löslichen und unlöslichen Quecksilberpräparaten zur Verwendung, und zwar wurden dieselben theils subkutan, theils intramuskulär dem Organismus einverleibt.

Wie bei jedem neuen therapeutischen Verfahren, so bedurfte es auch hier einer längeren Zeit der Beobachtung und Erfahrung, um eine Klärung $\mathrm{zu}$ schaffen. Auf die ersten hochgehenden Wogen der Begeisterung folgte bald eine starke Verflauung der Stimmung, die sogar bei einzelnen Autoren einer gänzlichen Verdammung der Methode gleichkam.

Wenn wir, was uns hier spezieller interessirt, nur auf die unlöslichen Präparate otwas näher eingehen - von einer Beurtheilung des Werthes dor Injektionstherapie im allgemeinen sehen wir ganz $a b-$, so verdient unter den Vorzügen derselben in erster Linie der grosse Quecksilbergehalt und die daraus resultirende Möglichkeit, mit wenigen Injektionen eine ausreichende Kur durchzuführen, hervorgehoben zu werden.

Unter den Nachtheilen der unlöslichen Salze, bezw. ihrer Verwendung - unwesentliche Nebenwirkungen lassen wir ganz ausser acht - rangirt an erster Stelle das Unvermögen unsererseits, im Augenblick, wo die Merkurialisation beginnt, diese $z u$ unterbrechen, und es sind daher auch, speziell im Anfang der Injektionsära, eine Reihe akuter Quecksilberintoxikationen mit den entsprechenden klinischen Symptomen und bei un-

1) Nach einem auf der 73. Versammlung Deutscher Naturforscher und Aerzte in Hamburg gehaltenen Vortrage. tiren, ob wir heute in Folge der verbesserten Technik in Stande sind, jede der erwähnten Inconvenienzen ganz zu vermeiden, resp. dieselben auf ein Minimum herabzudrücken, das darf man aber mit Fug und Recht behaupten: man ist vollständig ausser Stande, in dieser Beziehung eine Garantie zu übernehmen.

Von allen im Laufe der Jahre zur Injektion vorgeschlagenen unlöslichen Präparaten kommen - neben dem regulinischen Quecksilber - jetzt wohl nur noch Calomel vapore paratum, Hydrargyıum oxydatum flavum, Hydrargyrum thymolicum und Hydrargyrun salicylicum zur Verwendung, von denen zweifellos das letztere siclı der grössten Verbreitung erfreut.

Eine unsererseits gemachte, hierher gehörige klinische Beobachtung. deren Vorkommen wir bereits aut dem diesjährigen Congress der Deutschen del'natologischen Gesellschaft mittheilten, betrifft einen Fall von Gumnabildung an den Injektionsstellen von Hydrargyrum salicylicum; wir glauben aber von vornherein betonen $\mathrm{zu}$ sollen, dass wir weit davon entfernt sind, aus der einen klinischen Wahrnehmung irgendwelche verallgemeinernden Schlüsse zu ziehen, glauben aber immerhin, dass der Fall in mehrfacher Beziehung der Erwähnung werth ist.

Krankengeschichte: Im Monat März 1897 consultirte uns auf Veranlassung eines gynäkologischen Collegen der ca. 30 Jahre alte Kaufmann X., da seine Frau im achten Monate der Gravidität ein faultodtes, hereditär-syphilitisches Kind geboren hatte. Anamnestisch liess sich eruiren, dass der zur Zeit blühend aussehende, kräftige Patient im November 1895 , also reichlich $1 \frac{1}{4}$. Jahre vorher, in Russland kurze Zeit an einem sehr schnell verheilenden Ulcus des Penis ärztlich behandelt, und da keine weiteren Krankheitserscheinungen von ihm bemerkt waren, im Juli 1896 die Ehe eingegangen war. Bald nach seiner Verheirathung sah er sich veranlasst, wegen intensiver Halsbeschwerden einen Laryngologen aufzusuchen, der - nach persönlichen Mittheilungen - auf Grund von Plaques muqueuses an der hinteren Rachenwand, auf den Tonsillen, an der Zunge und der Lippe die Diagnose Lues stellte und den Patienten neben interner Jodkali- und lokalätzender Therapie während 7-8 Wochen mit $10-12$ in die beiderseitige Glutäalmuskulatır applizirten Hydrargyrum salicylicum-Injektionen (Hydrargyrum salicylicum 1.0, Paraffinum liquid. 10,0) in der allgemein üblichen Weise behandelte. Der lokale Effekt der letzteren wal der, dass der Patient einige Tage lang Schmerzen verspürte, im übrigen aber seinem Berufe ruhig nachgehen konnte.

Bei der ersten im März 1897 unsererseits vorgenommenen Untersuchung waren im wesentlichen nur ausgesprochene syphilitische Plaques auf beiden Tonsillen sowie reichlich pigmentirte Stellen am Stamm nachweisbar; in der beiderseitjgen Glutäalmuskulatur, und zwar im mittleren Theile derselben, liessen sich mehrere derbe, schmerzlose Knoten deutlich palpiren, ein Befund, auf den wir unseres Wissens bei Aufnahme der Anamnese vom Patienten selbst aufmerksam gemacht wurden und der uns neben anderen Gründen veranlasste, demselben ebenso wie es der überweisende Arzt auch bereits gethan hatte, eine Inunktionskur vorzuschlagen.

Unter einer über vier Wochen sich erstreckenden, durch einen geschulten Krankenwärter mit Unguentum Hydrargyri cinereum ausgefïhrten Behandlung und gleichzeitiger lokaler Therapie mittels Sublimat in alkoholisch-ätherischer Lösung trat bald Heilung ein, und im Laufe des Jahres hatten wir mehrfach Gelegenheit, uns von dem Wohlbefinden des Patienten zu überzeugen. Trotzdem entsprachen wir seinem Wunsche nach Wiederholung einer Kur mit Rücksicht auf spätere Nachkommenschaft und liessen ihn etwa ein halbes Jahr nach 
der ersten Inunktionckur eine zweite solche von etwa dreiwöchentlicher Dauer vornehmen.

Trotz der Anweisung, sich hie und da vorzinstellen, entzog sich der Patient unserer Beobachtung, und erst Ende April 1898, also fiinf Monate nach Beendigung der zweiten Einreibungskur, stellte er sich mit fraglichen Reizerscheinungen im Halse vor, die aber ohne weitere Maassnahmen auf Salzwassergurgelungen und Rauchverbot verschivanden.

Es verging dann ein weiteres Jahr, ohne dass wir vom Patienten etwas hörten. Im Mai 1899 führten ihn mehrfache Krankheitserscheinungen zu uns: zahlreiche derbe, schuppende Papeln auf der Glans Penis, nässende Papeln am Skrotum und mehrere grössere Pnstelnsyphilide an beiden Unterschenkeln.

Abgesehen von lokaler Behandlung mit Calomel, resp. Quecksilberpflastermull, verordneten wir mit Rücksicht auf den Charakter der Erscheinungen an den unteren Extremitäten intern Jodkalium und eine mehrwöchentliche Inunktionskur, Maassnahmen, unter denen sehr bald eine Heilung aller symptome erfolgte.

Wiederum entschwand nun der Kranke, da er vielfach auf Reisen im Auslande sich befand, für läıgere Zeit unserer Beobachtıng. Als er im Dezember 1899, und zwar krank zu uns kam, gab er an, innerhalb der letzten 3-4 Wochen neben allmählich zunehmenden Beschwerden beim Gehen und Sitzen ein Stärkerwerden der linken Hinterbacke, die seit kurzem auch nässe, wahrgenommen zu haben. Objektiv war folgendes zu constatiren:

Die mittlere Partie der linken Hinterbacke ist geschwollen, leicht geröthet und höher als normalerweise temperirt. Bei der Palpation lässt sich ein derb-elastischer, gegen die Ungebung scharf abgesetzter, auf Druck nicht iibermässig empfindlicher, einheitlicher Tumor nach weisen, dessen Höhen- und Breitendurchmesser ca. 10, dessen Tiefendurchmesser reichlich $5 \mathrm{~cm}$ beträgt. Im Bereiche der den Tumor bedeckenden Haut, die hier prall gespannt und unverschieblich ist, sind drei, annähernd pfenniggrosse, scharf umgrenzte Hautdefekte vorhanden. deren Grund mit einem speckigen Belag bedeckt ist und aus denen sich auf Druck eine minimale, dünne, molkenähnliche Fliissigkeit, untermischt mit einigen grau-gelblichen, bröckligen Massen anlsdriicken lässt.

Bei der Untersuchung der sonst normal erscheinenden rechten Hinterbacke sind einige kleine, schon bei der ersten Consultation constatirte isolirte Knoten, harte Infiltrationsheerde in der Glutäalmuskulatur zu fühlen, die an diesen Stellen mit der Haut verlöthet ist.

Da wir auf Grund des klinischen Gesammtbildes ein in der. linksseitigen Glutäalmuskulatur sitzendes solitäres Gumma init beginnender Erweichung und spontanem Durchbruch an einigen in die Gummabildung miteinbezogenen Stellen der Hautbedeckung annahmen, so verordneten wir neben körperlicher Ruhe lokal Hydrargyrumparaplast, Sitzbäder und innerlich reichlich Jodkalium. Bereits nach zehn Tagen war eine deutliche Veränderung. der Grösse und Consistenz des Tumors nachweisbar, und der weitere Rïckgang desselben stand in einem auffallenden Missverhältniss zu der geringen Sekretion aus den perforirten Stellen, von denen zwei während der ersten Zeit der Behandlung durch Einschmelzung der sie trennenden Hautbrücke zu einem grösseren Defekt sich umgewandelt hatten, während die dritte für sich bestehen geblieben war.

Nach etwa einmonatlicher Behandlung konnte der Patient mit dem Resultate entlassen werden, dass die offenen Stellen verheilt, auch alle sonstigen Krankheitserscheinungen verschwunden waren und die Geschwulst einer centralen trichterförmigen, narbigen Einziehung Platz. gemacht hatte.

Nach seiner Genesung ging Herr X. wiederum ins Ausland, und wir hörten nichts von ihm, bis er Ende Juni 1900, also ein halbes Jahr später in der Sprechstunde erschien. Er war, um sich in der Heimath ärztlich behandeln zu lassen, von der Pariser Weltausstellıng zurückgekehrt, wo er im Auftrage des von ihm vertretenen Geschäftshauses unter grossen körperlichen Strapazen und Entbehrungen bezïglich der Verpflegung die letzten Monate verbracht hatte.

Einmal klagte er iiber ziemlich intensive Schlingbeschwerden, als deren Ursache die lokale Untersuchung einen an der hinteren Rachenwand sitzenden, reichlich bohnengrossen, von scharfen Rändern begrenzten Defekt mit schmntzig-speckigem Grund innerhalb gerötheter und geschwollener Schleimhaut ergab. Sodann bot die rechte Hinterbacke fast dasselbe Bild dar, wie es oben an der linken beschrieben wurde, nur mit dem Unterschiede, dass hier die bedeckende Haut noch vollkommen intakt war und der infiltrirende Tumor noch nicht so bedeutende Dimensionen angenommen hatte.

Die Halsaffektion, von nns als ulzerirtes Gumma der hinteren Rachenwand gedeutet, wurde nun sofort lokal behandelt, ebenso die gleichen örtlichen therapeutischen Maassnahmen wie bei der linksseitigen Muskelerkrankung inszenirt, neben der internen Jodtherapie aber gleichzeitig eine energische Inunktionskur begonnen. $\mathrm{Da}$ die Halsbeschwerden bald verschwanden, auch die Glutäalinfiltration zuzückging, so kehrte der Patient nach etwa dreiwöchentlicher Behandlung, ohne indessen als geheilt entlassen zu sein, auf Drängen seines Geschäftshauses nach Paris zuruick. Während der nun folgenden Zeit nahı er, da sehr bald sich wieder Krankheitssymptome in der Glntäalmuskulatur einstellten, indessen nicht regelmässig, Jodkali, das ihm stets auch bald Erleichterung verschaffte, ein. Zehn Wochen nach Unterbrechung der Behandlung bei uns sahen wir ihn wieder und konnten nun an der erkrankten Stelle einen Tumor von ca. $7 \mathrm{~cm}$ Höhe und $5 \mathrm{~cm}$ Tiefe und Breite constatiren, dessen Hautbedeckung jetzt bereits auch an einigen Stellen perforirt war und Sekretion darbot, Erscheinungen, welche auf eine mehrwöchentliche erneute Jodbehandlung und eine wiederholte Inunktionskur so weit wichen, dass in der Umgebung einer starken centralen Einziehung der Haut eine etwa kleinapfelgrosse, im Muskel gelegene, absolut schmerzlose Verdickung zurückblieb. Da ausserdem auch alle subjektiven Beschwerden fehlten, so brach der Patient die Behandlung ab, sollte sich indessen bald in seiner Hoffnung, nun definitiv geheilt zu sein, durch den weiteren Verlauf bitter getäuscht sehen.

Als er Mitte Juni dieses Jahres zu uns kam, berichtete er, dass kaum einige Wochen nach der letzten Untersuchung unsererseits die rechte Hinterbacke allmählich wieder stärker geworden und daran anschliessend Nässen aus der vorher verheilten narbigen Einziehung aufgetreten sei; er habe dann neben heissen Bädern im Laufe der Monate März und April intermittirend Jodkali - und zwar stets mit positivem Erfolg - im ganzen etwa $100 \mathrm{~g}$ genommen, seit 4-6 Wochen aber mit jeder Behandlung ansgesetzt.

Objektiv war oberhalb der narbigen Einziehung, indessen in $\mathrm{Zn}$ sammenhang mit derselben in der centralen Glutäalpartie eine ca. $4 \mathrm{~cm}$ breite und ebenso hohe schmerzlose, derbe Infiltration nachweisbar; eine eingetrocknete Kruste bedeckte eine der frïheren, wieder aufgebrochenen Perforationsöffnungen.

Der Patient, welcher trotz eindringender, wiederholter Zurede bisher niemals zu einer Zittmannkur oder einer anderweitigen klinischen Behandlung zu bewegen gewesen war, entschloss sich nun wenigstens, eine von ins bis jetzt leider immer unterlassene Punktion des Tumors bei sich vornehmen zu lassen. Wir gingen mit einer dicken Punktionsnadel mitten in den Tumor ein und kratzten, da hierbei wenig Material gewonnen wurde, mit einem durch die erweiterte Punktionsöffnung eingefïhrten kleinen, scharfen Löffel Muskelmassen ans. Die vom Centrum und Grunde der Geschwulst stammenden Gewebstheile erwiesen sich mikroskopisch als Detritıs und fettig metamorphosirte Zellen; eine chemische, durch Herrn Dr. phil. Drenckmann gütigst ausgeführte Untersuchung ergab die Gegenwart von Hydrargyrum.

Ein zur Entnahme weiteren, für eine eingehendere mikroskopische und chemische Untersuchung bestimmten Materials unsererseits vor geschlagener chirn'gischer Eingriff, der zweifellos auch aus therapeutischen Riicksichten angezeigt gewesen wäre, wurde vom Kranken entschieden abgelehnt, und wir mussten uns daher wiederum mit einer Inunktionskur neben gleichzeitiger Jodmedikation bescheiden, therapentische Maassnahmen, welche innerhalb etwa sechs Wochen einen Ruckgang aller Erscheinungen erkennen liessen.

Jedoch besteht auch heute noch eine mässige, im Muskel oberflächlich relegene Infiltration, sodass wir die Möglichkeit eines nochmaligen Rezidivs, resp. erneuten Aufflackerns des Prozesses nicht für gänzlich ausgeschlossen halten.

Schliesslich sei noch erwähnt, dass die Gattin unseres Kranken, welche nach der ersten Fehlgeburt sehr schwere luetische Allgemein erscheinungen darbot, trotz mehrfacher energischer Behandlung des öfteren von Rezidiven heimgesucht wurde, auch noch zweimal, und zwar in friihen Monaten abortirte, vor einem viertel $J_{\text {ahr }}$ einem iiber acht Pf $m$ n schweren, bisher gesunden Kinde das Leben geschenkt hat.

Wenn wir jetzt anf einzelne Punkte ans der Krankengeschichte noch näher eingehen und unter Würdigung derselben unsere Befunde zu deuten versuchen, so verdient zunächst wohl die Thatsache hervorgehoben zu werden, dass es in unserem Falle noch nach Verlauf von vollen fünf Jahren gelang, Reste der früheren Hydrargyrum salicylicum-Injektionen in der Muskulatur in überzeugender Weise auf chemischem Wege nachzuweisen. Leider mussten wir uns aus den oben schon erwähnten Gründen mit einer einmaligen qualitativen Untersuchung begnügen.

Soweit uns die Litteratur zur Verfügung stand, war es nicht möglich, eine bezüg!iche, auf eine annähernd gleich lange Zeit sich erstreckende Angabe ausfindig $z u$ machen. Zwar ist es ja allgemein bekannt, dass einige Quecksilberpräparate, so unter anderen Oleum cinereum, Calomel, wochen- und monatelang post injektionem unresorbirt bleiben können, aber Wolters (1), der in mehreren Monographieen sich mit den lokalen Veränderungen nach intramuskulärer Injektion von salicylsaurem Quecksilber spezieller beschäftigt hat, giebt ausdrücklich an, dass in seinen Fällen, wo die Einspritzungen das eine Mal drei Monate, das andere Mal die letzte zwei, die älteste $5 \frac{1}{2}$ Jahre zurücklag, ein Nachweis von Hydrargyrumresten nicht gelang. Wenn er daher auf Grund dieser seiner Untersuchungen zu dem Resultate kommt: 
"dieser Befund ist wohl geeignet, den Vorwurf zu entkräften, dass die Depottherapie auf lange Zeit hinaus noch Gefahren in sich berge durch eventuelle plötzliche Resorption von Quecksilber aus alten Depots“, so bedarf diese Schlussfolgerung nach unserer Beobachtung doch wohl einer gewissen Einschränkung, auch wenn mit Wahrscheinlichkeit anzunehınen ist, dass es sich hiel um abgekapselte und dadurch der Resorption entzogene Heerde handelt.

Eine nähere Erörterung der eben nur gestreiften Frage würde uns zu weit führen, vielleicht dürfte es sich aber empfehlen, auf diesen Punkt: die Möglichkeit des Nachweises von Quecksilber in alten Injektionsschwielen, für die Zukunft wieder mehr das Augenmerk zu richten, und wir zweifeln nicht, dass derartige Fälle, wenn sich auch nicht grade häufen, so doch keinesfalls vereinzelt bleiben werden. Denn, wenn man, wie wir es jetzt regelmässig thun, jahrelang bei allen mit Einspritzungen behandelten $\mathrm{Pa}-$ tienten eingehender darauf achtet, wird man - in Uebereinstimmung mit Wolters Infiltrate, resp. knotige Indurationen als Residuen früherer Quecksilbereinspritzungen - von wem sie auch immer gemacht sein mögen - als einen mehr denn ausnahmsweisen Befund registriren können. Wir möchten diese Frage nur kurz berührt haben, da dieselbe erst wieder in neuester Zeit seitens Blaschko's (2) in wesentlich günstigerem Sinne behandelt ist.

Wie wir ferner schon oben kurz ausführten, sind wir geneigt, die beschriebenen pathologischen Veränderungen der Gesässmuskulatur als solitäre Gummata syphilitica zu deuten. Ist dem auch so, oder ist die Affektion als eine Erweichung chronischentzündlicher, auf die Hydrargyrum salicylicum-Injektionen zurückzuführender Infiltrate aufzufassen?

Wir wollen einmal annehmen, es handle sich um einen nicht mit Einspritzungen behandelten Syphilitiker. Derselbe komme wie unser Patient vier Jahre nach seiner Infektion zu uns mit dem gleichen allmählich, innerhalb mehrerer Wochen, ohne prodromale und synchrone Allgemeinerscheinungen und unter mässigen subjektiven Beschwerden entstandenen Muskeltumor, bei dem neben lokaler Quecksilberpflasterbehandlung das eine Mal auf eine interne Jodmedikation, das andere Mal auf gleichzeitige Inunktionen eine Rückbildung erfolgt mit Hinterlassung eines Defektes im betroffenen Gewebe - würde da irgend Jemand ein Bedenken tragen, ein Gumma. syphiliticum $\mathrm{zu}$ diagnostiziren? Wir meinen: Nein.

Und auch die Thatsache, dass nach Abheilung des linksseitigen Krankheitsheerdes, also nach vorhergehender erfolgreicher antiluetischer Allgemeinbehandlung, der gleiche Prozess auf der anderen Seite, und zwar gleichzeitig mit einem erweichten Gumma der hinteren Rachenwand sich einstellte, spricht keinesfalls gegen die Annahme eines gummösen Prozesses in beiden Fällen, da wir ja wissen, dass trotz regelrechter Behandlung in kürzeren oder längeren Intervallen mehrere tertiäre Ausbrüche nicht nur an verschiedenen Orten, sondern auch an der gleichen Stelle auf einander folgen können.

Die einzig mögliche, differentiell-diagnostisch in Betracht zu ziehende Annahme, dass es sich im vorliegenden Falle nicht um Gummata, sondern um Abszedirung chronisch-entzündlicher, auf die vor Jahren vorgenommenen Injektionen zurückzuführender Infiltrationsheerde handle, ist nicht ohne weiteres von der Hand zu weisen, zumal wir, wie eingangs schon kurz erwähnt wurde, ja wissen, (4), (5), dass unlösliche Quecksilberpräparate eventuell monatelang unresorbirt in loco applicationis liegen bleiben und Erweichungsheerde bedingen können; ferner die histologischen Untersuchungen von Wolters dargethan haben, welche bedeutenden Veränderungen noch nach mehreren Jahren in palpatorisch nachweisbaren, im menschlichen Muskel nach Hydrargyrum salicylicum-Einspritzungen zurückgebliebenen Indurationen vorhanden sein können, ohne dass in vita funktionelle Störungen bestanden hatten.

Immerhin halten wir in unserem Falle diese Möglichkeit der Erklärung für ausgeschlossen, glauben vielmehr, der günstige, deutlich wahrnehmbare Erfolg der Jodbehandlung auf der einen und der Jod- und Quecksilberbehandlung auf der anderen Seite ist als das wesentlichste Moment zur Unterstütung unserer Diagnose „Gumma“ heranzuziehen.

Einem hiergegen möglicherweise $\mathrm{zu}$ erhebenden Vorwurfe möchten wir von vornherein begegnen. Es ist uns ja bekannt, dass Quecksilberpflaster nicht nur syphilitische, zul Erweichung neigende pathologische Prozesse im erweichenden Sinne günstig beeinflusst, und man könnte daher geneigt sein, nicht der Jodmedikation als vielmehr dem Pflaster den wesentlichsten, aber dann nicht spezifischen Effekt in unserem Falle zuzuschreiben. Gegen diese Annahme spricht aber unserer Ueberzeugung nach das auffallende Missverhältniss zwischen der Menge des sezernirten Sekretes und dem Rückgange des Tumors.

Wenn wir daher auch unsere Auffassung als zu Recht bestehend voraussetzen, d. h. die fragliche Affektion als ein tertiärsyphilitisches Produkt ansehen, so spielen nach unserer Meinung die Hydrargyrum salicylicum-Injektionen, deren Applikationsstelle ja mit dem Sitz der späteren Gummata identisch ist, trotzdem eine gewisse Rolle bei der Genese der Gummata.

Im grossen und ganzen kann man, was die Aetiologie derselben anbetrifft, nach Haslund (6), Marschalkó (7), Neumann (8), Neisser (9) u. s. w. für die Mehrzahl der Fälle heute wohl die Anschauung als allgemein anerkannt annehmen, dass neben constitutionellen Leiden, chronischem Alkoholismus, schlechten Lebensverhältnissen eine Hauptursache der Entstehung der tertiären Syphilis eine zu spät eingeleitete erste Kul oder ungenügende, resp. ganz unterlassene merkurielle Behandlung in den ersten, unmittelbar auf die Infektion folgenden Stadien ist. Des weiteren wissen wir, dass die tertiären gummösen Formen - einem auch sonst öfter in der Pathologie beobachteten Gesetze folgend - sich im allgemeinen gern an einem auch anderweitig lädirten, weniger widerstandsfähigen Organ lokalisiren, im speziellen sich mit Vorliebe an solchen Stellen etabliren, welche im primären und sekundären Stadium Sitz syphilitischer Krankheitsheerde waren, und zwar spielen bei ihrer Entstehung Traumen, sei es mechanischer, chemischer oder thermischer Natur als occasionelle Ursache eine hervorragende Rolle (10), (11), (12).

Unter Berücksichtigung dieser Anschauungen lässt sich die Entstehung der Glutäalgummen in unserem Falle vielleicht am ungezwungensten so erklären:

Die Hydrargyrum salicylicum-Injektionen haben am Orte der Applikation entzündliche Erscheinungen hervorgerufen und im Muskelgewebe chronische Veränderungen, loci minoris resistentiae gesetzt, welche das Substrat für die späteren tertiären Krankheitsheerde abgegeben haben. Als veranlassendes Moment für die Etablirung des Tertiarismus überhaupt sind bei dem keinesfalls dem Alkohol ergebenen Patienten wohl in erster Linie die ausserordentlich spät eingeleitete primäre spezifische Kur und das durch seinen Reiseberuf bedingte unregelmässige und strapaziöse Leben anzuschuldigen. $\mathrm{Ob}$ überhaupt eine und bejahendenfalls welche lokale, die Gummabildung anregende äussere Schädigung hier in Betracht zu ziehen ist, lässt sich nicht mit Bestimmtheit entscheiden. Da aber kein Trauma, etwa ein Schlag oder Stoss anamnestisch nachweisbar war, könnte man eventuell daran denken, als solche die andauernden Erschütterungen und den auf die Glutäen ausgeübten Druck während tagelanger Eisenbahnfahrten anzusehen.

Mit Hilfe dieses supponirten äusseren schädigenden Momentes liesse sich vielleicht auch am ehesten die Thatsache erklären, dass, obwohl nach Virchow (13) die Glutäen mit unter die frequent gummatös erkrankten breiten Muskeln zu zählen sind, so verschwindend wenige Erkrankungen tertiären Charakters in den Glutäen bisher zu verzeichnen sind, trotz der enormen Anzahl von Quecksilberinjektionen an dieser Stelle. Immerhin würde aber auch bei dieser Interpretation noch die Thatsache der Aufklürung bedürfen, dass, obwohl doch zeitlich und lokal die gleichen Bedingungen vorlagen, erst die eine, dann nach Ablauf einiger Zeit die andere Seite gummatös erkrankte.

Bei diesem Versuch der Auslegung haben wir bisher ganz einen Faktor ausser Acht 'gelassen, der auch, vielleicht sogar in erster Linie in ätiologischer Beziehung in Betracht zu ziehen ist, nämlich den unsererseits erhobenen Befund von Quecksilber i m rechtsseitigen gummösen Heerde, ein Moment, das uns in den Stand setzt, auch ohne Zuhilfenahme eines äusseren schädigenden Einflusses den Vorgang der Gummabildung zu erklären.

Ohne der Theorie Zwang anzuthun, können wir uns vorstellen, dass das jahrelang im Muskel liegende Quecksilber wie ein Fremdkörper einen chronischen Reiz auf seine Umgebung ausgeübt und so die Veranlassung zur Etabli- 
rung des dulch allgemeine Verhältnisse begiinstigtell Tertiarismus abgegeben hat.

Obwohl wir ja links den Nachweis von Quecksilbel zu führen versäumt hatten, lässt sich, wenn man die vorstehende Theorie acceptirt, der verschiedene Verlauf des Gummas auf der linken und rechten Seite am besten so denken, dass auf der ersteren, wo eine deutliche centrale Erweichung des Tumors mit Perforation der Haut, und zwar gleich beim ersten Auftreten vorlag. alles Metall nach aussen entleert, alsgestossen wulde, daher die Veranlassung für weitere gummöse Gewebsproliferation fehlte, hingegen rechts, wo die Erkrankung sich nach voraufgegangener spezifischer Behandlung einstellte und in einem weit frïheren Stadium therapentische Maassuahmen inszenirt wurden, eine Weiterentwickelung des Prozesses, vor allem eine weitgehende Erweichung hintangehalten wurde, sodass auch hellte noch die definitive Heilung zweifelhaft orscheint. ${ }^{1}$ )

Obwohl des Ferneren eine auf lange Zeit sich erstreckende Entwickelıng von Muskelgummen nichts Ungewöhnliches ist (14), muss in unserem Falle auf der rechten Seite der eigenthümliche Verlauf der Erkrankung wohl auch mit anf den Umstand zurückgeführt werden, dass der Patient sich niemals die für eine definitive Heilung erforderliche Zeit und Schonıng gönnte, sondern imıner, sobald ein Rückgang eintrat, aussetzte.

Litteratur. 1. M. Wolters, Ueber die lokalen Veränderungen nach intramuskulärer Injektion unlösliclıer Quecksilberpräparate. Archiv für Dermatologie 1895, Bd. XXXII. - M. Wolters, Ueber lokale Veränderungen nach intramuskulärer Injektion von Hydrargyrum salicylicum. Archiv für Dermatologie 1897, Bd. XXXIX. - 2. A. Blaschko, Ueber einige Grundfragen bei der Behandlung der Syphilis. Berliner klinische Wochenschrift 1901, No. 2 und 3. - 3. M. Wolters, siche oben. - 4. Siehe M. Kaposi, Pathologie und Therapie der Syphilis Stıttgart 1891, F. Enke. S. 468. - 5. M. Wolter's, siehe oben. 6. A. Haslund, Ueber die Entstehung der tertiären Syphilis. Monatshefte für praktische Dermatologie 1893, Bd. XVI. - 7. Th. v. Marsclialkó, Beiträge zur Aetiologie der tertiäreı Lues, insbesondere iiber den Einfluss der Quecksilberbehandluno auf das Auftreten tertiärer Symptome. Archiv für Dermatologie 1891, Bd. XXIX. 8. J. Ne umann, Syphilis, Wien 1899, A. Hölder. - 9. A. Neisser, Ueber die ätiologischen Bezichungen der tertiären Lues zur Therapie in der Frühperiode. Verhandlungen der Deutschen dermatologischen Gesellscliaft, 5. Congress, Wien und Leipzig 1886, W. Braumüller. 10. Bäumler, Syphilis. Ziemssen's Handbuch der speziellen Pathologie und Therapie, Leipzig 1886, F. C. W. Vogel. - 11. E. Lang, Vorlesungen über Pathologie und Therapie der Syphilis. Wiesbaden 1884-1886, J. F. Bergmann. - 12. E. Lesser, Lehrbucl der Haut und Geschlechtskrankheiten. Leiprig 1897, F. C. W. Vogel. - 13. Virchow, Die krankhaften Gesclwwilste. - 14. Bier, Beitrag 7ur Kenntniss der Syphilome der äusseren Muskulatur. Mittheilungen der chirurgisehen Klinik zu Kiel, 1888. 